\title{
DIRAC OPERATOR ON THE STANDARD PODLEŚ QUANTUM SPHERE
}

\author{
LUDWIK DĄBROWSKI \\ Scuola Internazionale Superiore di Studi Avanzati \\ Via Beirut 2-4, I-34014 Trieste, Italy \\ E-mail:dabrow@fm.sissa.it
}

ANDRZEJ SITARZ

Institute of Physics, Jagiellonian University, Reymonta 4, 30-059 Kraków, Poland E-mail: sitarz@if.uj.edu.pl

\begin{abstract}
Using principles of quantum symmetries we derive the algebraic part of the real spectral triple data for the standard Podleś quantum sphere: equivariant representation, chiral grading $\gamma$, reality structure $J$ and the Dirac operator $D$, which has bounded commutators with the elements of the algebra and satisfies the first order condition.
\end{abstract}

1. Introduction. It is a prevailing common opinion that Connes' approach to noncommutative geometry [4] is in a sense incompatible with the (compact) quantum-group examples of $q$-deformations [9]. The main obstacle was the construction of an appropriate Dirac operator $D$, fulfilling the requirements postulated in [5]. In this paper we solve this problem for the standard Podleś quantum sphere [14] by finding a Dirac operator satisfying the 'algebraic' part of the axioms of real spectral triples. We simplify our considerations by making an additional assumption of symmetry with respect to the $\mathcal{U}_{q}(s u(2))$ action, thus pursuing the principles of construction of equivariant real spectral triples [15].

2. The standard quantum sphere and its symmetry. We recall the definition of the algebra $\mathcal{A}\left(S_{q}^{2}\right)$ of the standard Podleś quantum sphere as a subalgebra of the quantum $S U(2)$ function algebra $[14,16]$.

2000 Mathematics Subject Classification: Primary 58B34; Secondary 17B37.

Key words and phrases: noncommutative geometry, spectral triples, quantum spheres.

Research of the second author supported by the European Science Foundation travel grant.

The paper is in final form and no version of it will be published elsewhere. 
Let $q$ be a real number $0<q \leq 1$ and $\mathcal{A}\left(S U_{q}(2)\right)$ be a $*$-algebra generated by $a$, $a^{*}$ and $b, b^{*}$, which satisfy the following commutation rules:

$$
\begin{array}{ll}
b a=q a b, & \\
b^{*} a=q a b^{*}, & b b^{*}=b^{*} b, \\
a^{*} b=q b a^{*}, & a^{*} a+q^{2} b b^{*}=1, \\
a^{*} b^{*}=q b^{*} a^{*}, & a a^{*}+b b^{*}=1 .
\end{array}
$$

The algebra $\mathcal{A}\left(S_{q}^{2}\right)$ is isomorphic to the subalgebra generated by:

$$
\begin{aligned}
& B=a b, \\
& B^{*}=b^{*} a^{*}, \\
& A=b b^{*} .
\end{aligned}
$$

They obey the following relations:

$$
\begin{array}{ll}
A B=q^{2} B A, & A B^{*}=q^{-2} B^{*} A, \\
B B^{*}=q^{-2} A(1-A), & B^{*} B=A\left(1-q^{2} A\right) .
\end{array}
$$

The quantized algebra $\mathcal{U}_{q}(s u(2))$ has $e, f, k, k^{-1}$ as generators of the *-Hopf algebra, satisfying the relations:

$$
e k=q k e, \quad k f=q f k, \quad k^{2}-k^{-2}=\left(q-q^{-1}\right)(f e-e f) .
$$

The coproduct is given by:

$$
\Delta k=k \otimes k, \quad \Delta e=e \otimes k+k^{-1} \otimes e, \quad \Delta f=f \otimes k+k^{-1} \otimes f .
$$

The counit $\epsilon$, antipode $S$, and star structure are as follows:

$$
\begin{array}{lll}
\epsilon(k)=1, & \epsilon(e)=0, & \epsilon(f)=0, \\
S k=k^{-1}, & S f=-q f, & S e=-q^{-1} e, \\
k^{*}=k, & e^{*}=f, & f^{*}=e .
\end{array}
$$

From the usual Hopf algebra pairing between $\mathcal{U}_{q}(s u(2))$ and $\mathcal{A}\left(S U_{q}(2)\right)$ we obtain an action of $\mathcal{U}_{q}(s u(2))$ on $\mathcal{A}\left(S U_{q}(2)\right)$ given on generators by:

$$
\begin{array}{lll}
k \triangleright a=q^{\frac{1}{2}} a, & & k \triangleright a^{*}=q^{-\frac{1}{2}} a^{*}, \\
k \triangleright b=q^{\frac{1}{2}} b, & & k \triangleright b^{*}=q^{-\frac{1}{2}} b^{*}, \\
e \triangleright a=-b^{*}, & & e \triangleright a^{*}=0, \\
e \triangleright b=q^{-1} a^{*}, & & e \triangleright b^{*}=0, \\
f \triangleright a=0, & & f \triangleright a^{*}=q b, \\
f \triangleright b=0, & & f \triangleright b^{*}=-a .
\end{array}
$$

This action preserves the $*$-structure:

$$
h \triangleright\left(x^{*}\right)=\left((S h)^{*} \triangleright x\right)^{*}, \quad \forall h \in \mathcal{U}_{q}(s u(2)), x \in \mathcal{A}\left(S_{q}^{2}\right) .
$$


Hence we derive the action of $\mathcal{U}_{q}(s u(2))$ on the generators of the standard Podleś sphere:

$$
\begin{aligned}
& e \triangleright B=-\left(q^{\frac{1}{2}}+q^{-\frac{3}{2}}\right) A+q^{-\frac{3}{2}}, \\
& e \triangleright B^{*}=0, \\
& e \triangleright A=q^{-\frac{1}{2}} B^{*}, \\
& k \triangleright B=q B, \\
& k \triangleright B^{*}=q^{-1} B^{*}, \\
& k \triangleright A=A, \\
& f \triangleright B=0, \\
& f \triangleright B^{*}=\left(q^{\frac{3}{2}}+q^{-\frac{1}{2}}\right) A-q^{-\frac{1}{2}}, \\
& f \triangleright A=-q^{\frac{1}{2}} B .
\end{aligned}
$$

3. Equivariant representation of $\mathcal{A}\left(\boldsymbol{S}_{q}^{2}\right)$. As the next step we shall find a representation of the algebra $\mathcal{A}\left(S_{q}^{2}\right)$ on a Hilbert space $\mathcal{H}$, which is equivariant under the action of $\mathcal{U}_{q}(s u(2))$ defined in (9).

Definition 1. Let $V$ be an $A$-module and $H$ be a Hopf algebra. Also, let $V$ and $A$ be $H$-modules. We call $V$ an $H$-equivariant $A$-module if the following condition is satisfied:

$$
h(\alpha v)=\left(h_{(1)} \triangleright \alpha\right)\left(h_{(2)} v\right), \quad \forall h \in H, \alpha \in A, v \in V .
$$

Here we use Sweedler's notation for the coproduct of $H$ and $\triangleright$ for the action of $H$ on $A$. (In other words, $V$ is a module over the crossed (smash) product of $H$ and $A$.)

Definition 2. A bounded representation $\pi$ of $A$ on a Hilbert space $\mathcal{H}$ is called $H$ equivariant if there exists a dense linear subspace $V$ of $\mathcal{H}$ such that $V$ is an $H$-equivariant $A$-module and $\pi(\alpha) v=\alpha v, \quad \forall v \in V, \alpha \in A$.

We shall use the (known) representation theory of $\mathcal{U}_{q}(s u(2))$.

LEMMA $3([9])$. The irreducible finite dimensional representations of $\mathcal{U}_{q}(s u(2))$ are labeled by $l=0, \frac{1}{2}, 1, \ldots$ and they are given by

$$
\begin{aligned}
& f|l, m\rangle=\sqrt{[l-m][l+m+1]}|l, m+1\rangle, \\
& e|l, m\rangle=\sqrt{[l-m+1][l+m]}|l, m-1\rangle, \\
& k|l, m\rangle=q^{m}|l, m\rangle,
\end{aligned}
$$

where $m \in\{-l,-l+1, \ldots, l-1, l\}$ and for any number $x$

$$
[x]:=\frac{q^{x}-q^{-x}}{q-q^{-1}} .
$$

For a given $l$ we denote the representation space by $V_{l}$.

The representations above are $*$-representations of $\mathcal{U}_{q}(s u(2))$ with respect to the (hermitian) scalar product for which the vectors $|l, m\rangle$ are orthonormal.

We attempt now to construct a $\mathcal{U}_{q}(s u(2))$-equivariant representation of $\mathcal{A}\left(S_{q}^{2}\right)$ on the Hilbert space completion of a $\mathcal{U}_{q}(s u(2))$-equivariant $\mathcal{A}\left(S_{q}^{2}\right)$-module constructed as the direct sum of all representations $V_{l}$. A priori some multiplicities can occur but we make a simplifying assumption that this is not the case, guided by the picture of spinors on 
the classical (commutative) sphere. However, it may be easily verified that the results of Lemma 3 could be generalized to the case with multiplicities.

Lemma 4. $A \mathcal{U}_{q}(s u(2))$-equivariant representation of $\mathcal{A}\left(S_{q}^{2}\right)$ on the Hilbert space completion of $V_{0} \oplus V_{\frac{1}{2}} \oplus V_{1} \oplus \ldots$ must have the following form:

$$
\begin{gathered}
B|l, m\rangle=B_{l, m}^{+}|l+1, m+1\rangle+B_{l, m}^{0}|l, m+1\rangle+B_{l, m}^{-}|l-1, m+1\rangle, \\
B^{*}|l, m\rangle=\widetilde{B}_{l, m}^{+}|l+1, m-1\rangle+\widetilde{B}_{l, m}^{0}|l, m-1\rangle+\widetilde{B}_{l, m}^{-}|l-1, m-1\rangle, \\
A|l, m\rangle=A_{l, m}^{+}|l+1, m\rangle+A_{l, m}^{0}|l, m\rangle+A_{l, m}^{-}|l-1, m\rangle,
\end{gathered}
$$

where $A_{l, m}^{j}, B_{l, m}^{j}, \tilde{B}_{l, m}^{j}$ for $j=+, 0,-$, are constants.

Proof. We use the covariance property (10) on the algebraic direct sum of $V_{j}$. First, from the action of $k$ on $B$ we see that $B$ must increase the exponent $m$ of the eigenvalue of $k$ by 1 . Similar arguments show that $B^{*}$ decreases $m$ by 1 and $A$ does not change $m$. Therefore $B|l, m\rangle$ is a sum:

$$
B|l, m\rangle=\sum_{i} B_{i}|i, m+1\rangle
$$

where the sum runs over $i=0, \frac{1}{2}, 1, \frac{3}{2}, \ldots$ with finite number of nonzero coefficients.

Applying $f^{l-m+1}$ to both sides we obtain 0 on the left-hand side and a sum starting with $i=l+2$ on the right-hand side. By linear independence of all elements in this sum, we see that the sum over $i$ in (15) runs only up to $l+1$. (This does not eliminate yet the possibility of $i<l-1$.)

Using a similar argument, with $B^{*}$ instead of $B$, we observe that again the terms with $i>l+1$, in a corresponding sum, do not appear. Since we are looking for a $*$-representation of $\mathcal{A}\left(S_{q}^{2}\right)$, we conclude that in both expressions $i$ runs from $l-1$ to $l+1$, as stated in (12-14). From the sphere defining relations (3) it follows that this holds also for $A$.

It follows from (12-14) that we can separate the half integer spin representations from the integer spin representations. In the sequel we restrict ourselves only to the case of the Hilbert space $\mathcal{H}_{\frac{1}{2}}$ given by completion of the direct sum

$$
V=V_{\frac{1}{2}} \oplus V_{\frac{3}{2}} \oplus \ldots
$$

of all half-integer representations of $\mathcal{U}_{q}(s u(2))$, motivated by the classical picture of (chiral) spinors over $S^{2}$.

We proceed by applying $f$ on both sides of (12) to obtain the following recursion relations for the coefficients $B_{l, m}^{j}$ :

$$
\frac{1}{q} B_{l, m+1}^{j} \sqrt{[l-m][l+m+1]}=B_{l, m}^{j} \sqrt{[l+j-m-1][l+j+m+2]} .
$$

We solve them explicitly for each value of $m$ :

$$
\begin{aligned}
& B_{l, m}^{+}=q^{m} \sqrt{[l+m+1][l+m+2]} \alpha_{l}^{+}, \\
& B_{l, m}^{0}=q^{m} \sqrt{[l+m+1][l-m]} \alpha_{l}^{0}, \\
& B_{l, m}^{-}=q^{m} \sqrt{[l-m][l-m-1]} \alpha_{l}^{-},
\end{aligned}
$$

where $\alpha_{l}^{ \pm}, \alpha_{l}^{0}$ are yet undetermined functions of $l$. 
If we apply $e$ to (13), we obtain

$$
\begin{aligned}
\widetilde{B}_{l, m}^{+} & =q^{m-1} \sqrt{[l-m+2][l-m+1]} \alpha_{l+1}^{-}, \\
\widetilde{B}_{l, m}^{0} & =q^{m-1} \sqrt{[l+m][l-m+1]} \alpha_{l}^{0}, \\
\widetilde{B}_{l, m}^{-} & =q^{m-1} \sqrt{[l+m][l+m-1]} \alpha_{l-1}^{+} .
\end{aligned}
$$

Finally, for $A$, using the action of $e$ on $B$ (or equivalently $f$ on $B^{*}$ ) we get:

$$
\begin{aligned}
& A_{l, m}^{+}=-q^{m+l+\frac{1}{2}} \sqrt{[l-m+1][l+m+1]} \alpha_{l}^{+} \\
& A_{l, m}^{0}=q^{-\frac{1}{2}} \frac{1}{1+q^{2}}\left([l-m+1][l+m]-q^{2}[l-m][l+m+1]\right) \alpha_{l}^{0}+\frac{1}{1+q^{2}}, \\
& A_{l, m}^{-}=q^{m-l-\frac{1}{2}} \sqrt{[l-m][l+m]} \alpha_{l}^{-} .
\end{aligned}
$$

Moreover, the $*$-representation condition yields a consistency relation between $\alpha^{+}$and $\alpha^{-}$:

$$
\alpha_{l+1}^{-}=-q^{2 l+2} \alpha_{l}^{+}, \text {for } l=\frac{1}{2}, \frac{3}{2}, \ldots .
$$

Now, we use the relations defining the algebra $\mathcal{A}\left(S_{q}^{2}\right)$ in order to calculate the form of $\alpha_{l}^{+}$and $\alpha_{l}^{0}$. The comparison of the $|l+1, m+1\rangle$ component of the action of the first relation in (3) on $|l, m\rangle$ gives the following recurrence relation in $l$ :

$$
\alpha_{l+1}^{0}[2 l+4]=\alpha_{l}^{0}[2 l]+\frac{1}{\sqrt{q}}\left(q-q^{-1}\right) .
$$

To get the relation for $\alpha_{l}^{+}$and obtain the initial terms (that is, values for $l=\frac{1}{2}$ ) we employ the last two relations in (3), of which we choose the following convenient combination:

$$
B^{*} B-q^{4} B B^{*}=\left(1-q^{2}\right) A .
$$

Our results can be summarized in the following lemma.

LEMMA 5. There are two inequivalent $\mathcal{U}_{q}(s u(2))$-equivariant representations of $\mathcal{A}\left(S_{q}^{2}\right)$ on the Hilbert space $\mathcal{H}_{\frac{1}{2}}$ given by formulae (12-14), (17-20) and by the following formulae for $\alpha_{l}^{+}$and $\alpha_{l}^{0}$ :

- $\pi_{+}$:

$$
\begin{array}{r}
\alpha_{l}^{0}=\frac{1}{\sqrt{q}} \frac{\left(q-\frac{1}{q}\right)\left[l-\frac{1}{2}\right]\left[l+\frac{3}{2}\right]+q}{[2 l][2 l+2]}, \\
\alpha_{l}^{+}=q^{-l-2} \frac{1}{\sqrt{[2 l+2]([4 l+4]+[2][2 l+2])}} . \\
\alpha_{l}^{0}=\frac{1}{\sqrt{q}} \frac{\left(q-\frac{1}{q}\right)\left[l-\frac{1}{2}\right]\left[l+\frac{3}{2}\right]-q^{-1}}{[2 l][2 l+2]}, \\
\alpha_{l}^{+}=q^{-l-1} \frac{1}{\sqrt{[2 l+2]([4 l+4]+[2][2 l+2])}} .
\end{array}
$$

- $\pi_{-}$:

Proof. By direct calculation with the help of the symbolic algebra program. ${ }^{1}$

${ }^{1}$ The source file with definitions and examples is available from the web-site: http://www.cyfkr.edu.pl/〜ufsitarz/maple.html 
First we verify the value of $\alpha_{\frac{1}{2}}^{+}$:

$$
\left(\alpha_{\frac{1}{2}}^{+}\right)^{2}=q^{-4 \mp 1} \frac{1}{([3])^{2}[4]},
$$

where the \pm signs corresponds to the freedom we have. This leads to the explicit solution of the recurrence relation (21), which is the formula (25). For $\alpha_{l}^{+}$we use two relations obtained from (22):

$$
\begin{gathered}
\left(\alpha_{l}^{+}\right)^{2} q^{2 l+3}[2 l+3][2]-\left(\alpha_{l-1}^{+}\right)^{2} q^{2 l+1}[2 l-1][2] \\
-\left(\alpha_{l}^{0}\right)^{2} q[4 l+2] /[2 l+1]+\alpha_{l}^{0} \sqrt{q}\left(q-q^{-1}\right)=0, \\
-\left(\alpha_{l}^{+}\right)^{2} q^{2 l+3}[4 l+6][2]+\left(\alpha_{l-1}^{+}\right)^{2} q^{2 l+1}[4 l-2][2] \\
+\left(\alpha_{l}^{0}\right)^{2} q([2])^{2}-\alpha_{l}^{0} \sqrt{q}\left(q-q^{-1}\right)[4 l+2] /[2 l+1]+\left(q-q^{-1}\right)^{2}=0 .
\end{gathered}
$$

By subtracting the left-hand sides to eliminate $\alpha_{l-1}^{+}$and using the result (25) for $\alpha_{l}^{0}$, we arrive at the final formula (26) presented above. Besides (22) we also check that the last two relations in (3) are separately fulfilled and that all elements of $\mathcal{A}\left(S_{q}^{2}\right)$ are represented as bounded operators on $\mathcal{H}_{\frac{1}{2}}$.

Note that the possible sign ambiguity in the formula for $\alpha_{l}^{+}$can be globally resolved by the redefinition of the basis.

4. Real spectral triple for $\boldsymbol{S}_{q}^{2}$. We begin by taking as the Hilbert space of Dirac spinors, on which $\mathcal{A}\left(S_{q}^{2}\right)$ is represented, the direct sum $\mathcal{H}:=\mathcal{H}_{\frac{1}{2}} \oplus \mathcal{H}_{\frac{1}{2}}$, with the representation given by:

$$
\pi(a)=\left(\begin{array}{ll}
\pi_{+}(a) & 0 \\
0 & \pi_{-}(a)
\end{array}\right)
$$

and the grading $\gamma$ :

$$
\gamma=\left(\begin{array}{ll}
1 & 0 \\
0 & -1
\end{array}\right) .
$$

It is obvious that the representation $\pi$ is bounded on $\mathcal{H}, \mathcal{U}_{q}(s u(2))$-equivariant and $[\pi(\alpha), \gamma]=0$ for all $\alpha \in \mathcal{A}\left(S_{q}^{2}\right)$.

The next ingredient we search for is the reality structure given by an antilinear operator $J$. Denote by $|l, m\rangle_{ \pm}$the orthonormal basis in the first (resp. second) copy of $\mathcal{H}_{\frac{1}{2}}$ in $\mathcal{H}$. We claim the following:

LEMMA 6. For all real $p>0$ the operator:

$$
J|l, m\rangle_{ \pm}=i^{2 m} p^{m}|l,-m\rangle_{\mp},
$$

satisfies $J^{2}=-1, \gamma J=-J \gamma$ and

$$
J \pi(\alpha) J \pi(\beta)=\pi(\beta) J \pi(\alpha) J, \quad \forall \alpha, \beta \in \mathcal{A}\left(S_{q}^{2}\right) .
$$

Proof. Since $m$ is fractional (hence $2 m$ is odd) and $J$ is antilinear, we have:

$$
\begin{aligned}
J^{2}|l, m\rangle_{ \pm} & =J\left(i^{2 m} p^{m}|l,-m\rangle_{\mp}\right) \\
& =-i^{2 m} p^{m} J|l,-m\rangle_{\mp}=-i^{2 m} p^{m} i^{-2 m} p^{-m}|l, m\rangle_{ \pm}=-|l, m\rangle_{ \pm} .
\end{aligned}
$$


The second property is obvious from the construction. The only nontrivial part is the verification of (32). We have verified this explicitly for two pairs $(\alpha, \beta)=(B, B)$ and $\left(B, B^{*}\right)$ of generators, which clearly suffices in view of the relation (22). Also, as an independent check, we have verified (32) with the help of a computer symbolic computation program for all the generators of the algebra.

REMARK 7 . Note that $J$ is not unitary unless $p=1$, in which case it has the same form as the classical charge conjugation on the sphere. However, only for $p=q, J$ is $\mathcal{U}_{q}(s u(2))$-equivariant, that is, for all $h \in \mathcal{U}_{q}(s u(2))$ :

$$
h J=J(S h)^{*} \text {. }
$$

In fact, it is the equivariance property $[15,12]$ which led us to this form of $J$.

Finally, we shall look for all possible Dirac operators $D$ which satisfy the following properties:

- $D$ is an (unbounded in general) selfadjoint operator with compact resolvent defined on a dense domain in $\mathcal{H}$ which contains $V \oplus V$,

- $D$ is $\mathcal{U}_{q}(s u(2))$-invariant, that is,

$$
D h=h D, \quad \forall h \in \mathcal{U}_{q}(s u(2)),
$$

- $D \gamma=-\gamma D$,

- the commutators $[D, \pi(\alpha)]$ are bounded for all $\alpha \in \mathcal{A}\left(S_{q}^{2}\right)$,

- $D J=J D$,

- $D$ satisfies the first order condition:

$$
[[D, \pi(\alpha)], J \pi(\beta) J]=0, \quad \forall \alpha, \beta \in \mathcal{A}\left(S_{q}^{2}\right) .
$$

The following is the main result of the paper.

THEOREM 8. All operators satisfying the conditions listed above are of the following form:

$$
D=\left(\begin{array}{ll}
0 & \bar{z} D_{-} \\
z D_{+} & 0
\end{array}\right), \text { where } D_{ \pm}|l, m\rangle_{ \pm}=\left[l+\frac{1}{2}\right]|l, m\rangle_{\mp} \text { and } z \in \mathbb{C} \backslash\{0\} .
$$

Proof. First, from the commutation with $\gamma$ we obtain that $D: \mathcal{H}_{ \pm} \rightarrow \mathcal{H}_{\mp}$. Then the equivariance forces that:

$$
D|l, m\rangle_{ \pm}=d_{l}^{ \pm}|l, m\rangle_{\mp},
$$

where $d_{l}^{ \pm}$are constants. In addition, the condition for $D$ to be hermitian yields

$$
d_{l}^{ \pm}=\overline{d_{l}^{\mp}} \text {. }
$$

Now, using the form of $J$ from (31) we calculate $D J-J D$ :

$$
\begin{aligned}
(D J-J D)|l, m\rangle_{ \pm} & =D i^{2 m} p^{m}|l,-m\rangle_{\mp}-J d_{l}^{ \pm}|l, m\rangle_{\mp} \\
& =i^{2 m} p^{m}\left(d_{l}^{\mp}-\overline{d_{l}^{ \pm}}\right)|l, m\rangle_{ \pm}=0 .
\end{aligned}
$$

Here we used (37) in the final step.

Next, as a consequence of the derivation property of commutators and of (32), the first order condition is satisfied for all $\alpha, \beta \in \mathcal{A}\left(S_{q}^{2}\right)$ iff it is satisfied for all pairs of generators. 
Furthermore, in view of (22), the Jacobi identity and skewsymmetry of commutators, it suffices to verify (34) just for two pairs of generators $(\alpha, \beta)=(B, B)$ and $\left(B, B^{*}\right)$. This yields first the following recurrence relation for $d_{l}^{+}$:

$$
d_{l}^{+}+d_{l+2}^{+}=[2] d_{l+1}^{+} \text {. }
$$

Hence,

$$
d_{l}^{+}=x q^{l}+y q^{-l}
$$

where $x$ and $y$ are arbitrary constants. Next, (34) also yields $x=-q y$, thus giving us the form of the Dirac operator as in (35), where $z$ is a complex parameter (we can rescale all $d_{l}^{+}$by the same $z$ and all $d_{l}^{-}$by $\bar{z}$ ). Using this form of $D$ we verify explicitly (again with the help of symbolic computation program) that the first order condition is satisfied for all pairs of generators of $\mathcal{A}\left(S_{q}^{2}\right)$.

The parameter $z$ must be nonzero in order for the resolvent of $D$ to be compact. It is also clear that the only accumulation points of the spectrum $\{ \pm|z|[l+1 / 2]\}$ of $D$ are $\pm \infty$ which guarantees the compactness of the resolvent. Finally, the Dirac operator $D$ is selfadjoint on the dense domain,

$$
\left\{\psi=\sum_{l, m, \sigma} c_{l, m, \sigma}|l, m\rangle_{\sigma}: c_{l, m, \sigma} \in \mathbb{C}, \sum_{l, m, \sigma}\left(1+|z|^{2}[l+1 / 2]^{2}\right)\left|c_{l, m, \sigma}\right|^{2}<\infty\right\},
$$

where the sum runs over $l=\frac{1}{2}, \frac{3}{2}, \ldots, m=-l, \ldots, l$ and $\sigma= \pm$.

For this form of $D$ we show that the commutators $[D, \pi(\alpha)]$, for the elements $\alpha$ of the algebra $\mathcal{A}\left(S_{q}^{2}\right)$ are bounded. To see this it suffices to check it only for the generators. We start with the generator $B$. For a given $l$, there are only three nonzero matrix elements ${ }_{-}\langle l+j, m+1|[D, B]| l, m\rangle_{+}$corresponding to $j=-1,0,1$. We verify that they are bounded. In the case $j=1$ we estimate:

$$
\begin{aligned}
& \left|-\langle l+1, m+1|[D, \pi(B)]| l, m\rangle_{+}\right| \\
& =q^{m} \frac{\sqrt{[l+m+1][l+m+2]}}{\sqrt{[2 l+2]([4 l+4]+[2][2 l+2]}} q^{-l-2}\left(\left[l+\frac{3}{2}\right]-q\left[l+\frac{1}{2}\right]\right) \\
& \leq C_{+} q^{m} q^{-l-m} q^{3 l} q^{-l} q^{-l}=C_{+},
\end{aligned}
$$

where $C_{+}$is a constant. Similarly, for $j=0$ :

$$
\begin{aligned}
& \left|-\langle l, m+1|[D, \pi(B)]| l, m\rangle_{+}\right| \\
& =q^{m} \frac{\sqrt{[l+m+1][l-m]}}{\sqrt{q}[2 l][2 l+2]}\left(q^{-1}-q\right)\left[l+\frac{1}{2}\right] \\
& \leq C_{0} q^{m} q^{-l} q^{4 l} q^{-l} \leq C_{0},
\end{aligned}
$$

for a suitable constant $C_{0}$. Finally, for $j=-1$ :

$$
\begin{aligned}
& \left|-\langle l-1, m+1|[D, \pi(B)]| l, m\rangle_{+}\right| \\
& =q^{m} \frac{\sqrt{[l-m][l-m-1]}}{\sqrt{[2 l+2]([4 l+4]+[2][2 l+2])}} q^{-l-2} q^{2 l}\left(\left[l+\frac{3}{2}\right]-q\left[l+\frac{1}{2}\right]\right) \\
& \leq C_{-} q^{m} q^{-l+m} q^{3 l} q^{-l} q^{-l} q^{2 l} \leq C_{-},
\end{aligned}
$$

where in the last line we have used $q^{-m} \leq q^{-l}$. It can be verified directly that similar bounds hold also for the generators $A$ and $B^{*}$, but this in fact is a consequence of (22) and the fact that $\pi$ is a bounded $*$-representation. 
Note that we get a perfect agreement with the picture of the classical spectral triple on the commutative sphere. Using the notation of $[2]$ (pp.407-419) we can (at $q=1$ ) identify $|l, m\rangle_{ \pm}$with $Y_{l, m}^{ \pm}$. Similarly, for $p=1$ our $J$ has the same form as the charge conjugation $C$ on spinors and one finds the eigenvectors and eigenvalues of $D$ to have the correct $q=1$ limit.

5. Conclusions. It is quite surprising that despite the earlier mentioned common belief that Connes' approach to noncommutative geometry does not cover quantum-group examples, we have been able to produce an example satisfying the algebraic axioms of real spectral triples (noncommutative manifolds). We have been informed by Alain Connes that also the dimension axiom can be satisfied (related to the asymptotic behavior of the eigenvalues of the Dirac operator). We believe that elaborating on this example will prove helpful to reconcile the noncommutative geometry with the $q$-geometry.

It should be noted that, unlike most of other approaches $[1,10,11,13]$, we do not assume at the beginning any form of the Dirac operator (apart from the requirement of equivariance) and we derive it step by step. Although its eigenvalues can be compared with, e.g., those in [1], the essential difference is the explicit realization of all the data on the Hilbert space. This includes the representation of the algebra and the proof that the resulting differential forms have bounded representation $[D, \pi(\alpha)]$, as well as a presentation of reality and chirality operations. Also, in comparison to [7, 3] (apart from using distinct starting algebras) our Hilbert space representation and the equivariance requirements are fundamentally different. Moreover, an important new ingredient is the reality operator.

There are still important problems to solve, which we shall address elsewhere: the analytic properties of the spectral triple (summability, regularity and finiteness) as well as the existence of the Hochschild cycle giving the volume form and Poincaré duality. (For details on these notions and their applications see [2], 10.5.) Last not least, we plan to study the details of the classical limit $q \rightarrow 1$, the relation of $\mathcal{H}_{ \pm}$and $\mathcal{H}$ to $q$-deformed Hopf bundles [8], and the equivariant Fredholm module obtained from our construction. Also the systematic analysis of other Podleś spheres (in particular, the equatorial quantum sphere) is in preparation [6].

Acknowledgments. The authors would like to thank P. M. Hajac for discussions and comments on the manuscript, and G. Landi for discussions. The first author acknowledges the financial support of the 'Geometric Analysis' Research Training Network HPRN-CT1999-00118 of the E. C. The second author would like to thank ESF for a travel grant and SISSA for hospitality.

\section{References}

[1] P. N. Bibikov and P. P. Kulish, Dirac operators on the quantum group $\mathrm{SU}_{q}(2)$ and the quantum sphere, Zap. Nauchn. Sem. POMI 245 (1997), Vopr. Kvant. Teor. Polya i Stat. Fiz. 14, 49-65, 283; translation in J. Math. Sci. (New York) 100 (2000), 2039-2050. 
[2] J. M. Gracia-Bondía, J. C. Várilly and H. Figueroa, Elements of Noncommutative Geometry, Birkhäuser, Boston, MA, 2001.

[3] P. S. Chakraborty and A. Pal, Equivariant spectral triples on the quantum SU(2) group, math.KT/0201004.

[4] A. Connes, Noncommutative Geometry, Academic Press, 1994.

[5] A. Connes, Noncommutative geometry and reality, J. Math. Phys. 36 (1995) 6194-6231.

[6] L. Dąbrowski, G. Landi, M. Paschke and A. Sitarz, in preparation.

[7] D. Goswami, Some noncommutative geometric sspects of $S U_{q}(2)$, math-ph/0108003.

[8] P. M. Hajac and S. Majid, Projective module description of the q-monopole, Comm. Math. Phys. 206 (1999), 247-264.

[9] A. Klimyk and K. Schmuedgen, Quantum Groups and their Representations, Springer, New York, 1998.

[10] K. Ohta and H. Suzuki, Dirac Operators on Quantum Two Spheres, Mod. Phys. Lett. A9 (1994), 2325-2334.

[11] R. Owczarek, Dirac operator on the Podles sphere, Int. J. Theor. Phys. 40 (2001), 163-170.

[12] M. Paschke, Über nichtkommutative Geometrien, ihre Symmetrien und etwas Hochenergiephysik, Ph.D. Thesis, Mainz, 2001.

[13] A. Pinzul and A. Stern, Dirac operator on the quantum sphere, Phys. Lett. B 512 (2001), 217-224.

[14] P. Podleś, Quantum spheres, Lett. Math. Phys. 14 (1987), 521-531.

[15] A. Sitarz, Equivariant spectral triples, this volume.

[16] S. L. Woronowicz, Twisted SU(2) group. An example of a noncommutative differential calculus, Publ. RIMS Kyoto Univ. 23 (1987), 117-181. 